Audiology

Neurotology
Audiol Neurotol 2012;17:381-385

DOI: $\underline{10.1159 / 000341815}$
Received: January 9, 2012

Accepted after revision: July 2, 2012

Published online: August 22, 2012

\title{
Heat Shock Protein 70 Gene Polymorphisms in Sudden Sensorineural Hearing Loss
}

\author{
Chen-Yu Chien ${ }^{a, b}$, Ning-Chia Chang ${ }^{c}$ Shu-Yu Tai ${ }^{a, d}$ Ling-Feng Wang ${ }^{b, e}$ \\ Ming-Tsang $\mathrm{Wu}^{\mathrm{a}, \mathrm{d}, \mathrm{f}}$ Kuen-Yao Ho $\mathrm{H}^{\mathrm{a}, \mathrm{b}, \mathrm{e}}$ \\ ${ }^{a}$ Graduate Institute of Medicine, College of Medicine, Kaohsiung Medical University, Departments of \\ b Otorhinolaryngology, ' Preventive Medicine, and d Family Medicine, Kaohsiung Medical University Hospital, \\ eDepartment of Otorhinolaryngology, School of Medicine, College of Medicine, Kaohsiung Medical University, \\ ${ }^{f}$ Graduate Institute of Occupational Safety and Health, Kaohsiung Medical University, and 9Department of \\ Otolaryngology, Kaohsiung Municipal Hsiao-Kang Hospital, Kaohsiung Medical University, Kaohsiung, Taiwan, ROC
}

\section{Key Words}

Genetic association • rs $2075800 \cdot$ Sudden deafness $\cdot$ HSP70

\begin{abstract}
Background: Heat shock proteins protect cells and tissues against different types of damage. Previous studies have revealed that the serum level of heat shock protein 70 (HSP70) increases in sudden sensorineural hearing loss (SSNHL) patients. We hypothesized that genetic variants of the HSP7O gene are associated with susceptibility to SSNHL. Methods: We conducted a case-control study with 160 SSNHL cases and 178 controls. Three tagging single nucleotide polymorphisms (SNPs) were selected. The genotypes were determined using TaqMan technology. Hardy-Weinberg equilibrium was tested for each SNP, and genetic effects were evaluated according to three inheritance modes. A haplotype analysis was also performed. Results: All three SNPs were in Hardy-Weinberg equilibrium. The CT genotype of rs 2075800 exhibited an adjusted odds ratio of 0.59 (95\% confidence interval 0.37-0.94; $p=0.027$ ). The T allele of SNP rs 2075800 was associated with SSNHL under the dominant model $(p=0.019$; odds ratio 0.59 ). Haplotype analysis of the three SNPs demonstrated that the haplotype TGC (rs2075800/rs1043618/
\end{abstract}

rs2763979) was statistically significant $(p=0.0137)$. Conclusions: These results suggest that HSP70 gene polymorphisms influence the susceptibility to the development of SSNHL in the Taiwanese population.

Copyright $\odot 2012$ S. Karger AG, Basel

\section{Introduction}

Sudden sensorineural hearing loss (SSNHL) is defined as a loss of at least $30 \mathrm{~dB}$ in 3 contiguous frequencies over a period of 3 days or less [Wu et al., 2006]. The incidence rates per 100,000 people in the Taiwanese population are 8.85 for men and 7.79 for women [Wu et al., 2006]. The etiology and pathogenesis of SSNHL are not known, but the proposed primary causes include viral infection, vascular disease, and autoimmunity [Wu et al., 2006]. SSNHL is a complex multifactorial disease that includes genetic factors, such as prothrombin G20210A, factor V Leiden G1691A, and methylene-tetrahydrofolate reductase (MTHFR) C677T [Schreiber et al., 2010].

The heat shock proteins are classified by molecular weight and grouped into families of different sizes [Macario and Conway de Macario, 2005]. These proteins

\section{KARGER}

Fax +4161306 1234

E-Mail karger@karger.ch

www.karger.com (c) 2012 S. Karger AG, Basel

$1420-3030 / 12 / 0176-0381 \$ 38.00 / 0$

Accessible online at:

www.karger.com/aud
Kuen-Yao Ho

Department of Otorhinolaryngology, Kaohsiung Medical University Hospital 100, Tzyou 1st Road

Kaohsiung 807, Taiwan (ROC)

Tel. +886 7312 1101, ext. 5009, E-Mail chenyu@ kmu.edu.tw 
function as intracellular chaperones which control protein folding and unfolding, and translocation under physiological conditions and following physical or chemical stress [Matouschek, 2003]. Extracellular heat shock proteins process and present immunogenic peptides to cytotoxic T cells, activate the innate immune system, and induce proinflammatory cytokine secretion from monocytes [Asea et al., 2000; Srivastava et al., 1994]. Heat shock protein 70 (HSP70) molecules play a key role in the immune response by functioning both as chaperones and inducers of proinflammatory cytokine secretion [Spagnolo et al., 2007].

SSNHL patients exhibit significantly higher levels of serum anti-HSP70 antibodies compared to normal controls [Park et al., 2006]. A previous study revealed a positive association between anti-HSP70 antibodies and positive outcomes in SSNHL [Gross et al., 2008]. Three members of the HSP70 gene family have been mapped on 6 p21.3 in the human leukocyte antigen (HLA) class III region [Milner and Campbell, 1990]. The human HSP70 gene family consists of HSPA1L (HSP70-Hom), HSPA1A (HSP70-1), and HSPA1B (HSP70-2) [Milner and Campbell, 1990]. The expression of HSPA1A and HSPA1B is heat-inducible, and these two genes encode an identical 641 amino acid protein. HSPA1L is not heat-inducible, and it encodes a protein that is highly related to HSPA1A [Milner and Campbell, 1990].

HSP70 gene polymorphisms may play a role in the pathogenesis of SSNHL. No data have been published on the relationship between $H S P 70$ genetic polymorphisms and the risk of SSNHL. This study investigated whether HSP70 gene polymorphisms are risk factors for SSNHL in Taiwanese subjects. We conducted a case-control study to systematically investigate the role of HSP70 tagging single nucleotide polymorphisms (tSNPs) in the development of SSNHL in the Taiwanese population.

\section{Materials and Methods}

\section{Subjects}

We recruited 160 patients with the SSNHL and 178 controls at the Kaohsiung Medical University Hospital between October 2010 and November 2011. The diagnostic criteria of SSNHL included sensorineural hearing loss of at least $30 \mathrm{~dB}$ in 3 contiguous frequencies using a pure tone audiogram with an onset within 3 days [Wu et al., 2006]. All the patients had no treatment for hearing loss or impairment before taking the hearing test. The exclusion criteria were hearing losses caused by acoustic neuroma, central lesions, Ménière's disease, multiple sclerosis, infectious diseases, drug-induced and noise-induced hearing losses, or hearing losses due to prior ear surgery.
Table 1. Baseline demographics of subjects

\begin{tabular}{lccc}
\hline & $\begin{array}{l}\text { Case } \\
(\mathrm{n}=160)\end{array}$ & $\begin{array}{l}\text { Control } \\
(\mathrm{n}=178)\end{array}$ & p value \\
\hline $\begin{array}{l}\text { Age, years } \\
\begin{array}{l}\text { Sex, } \mathrm{n}(\%) \\
\text { Male }\end{array}\end{array}$ & $51.6 \pm 15.0$ & $51.7 \pm 8.0$ & $0.917^{\mathrm{a}}$ \\
Female & $86(54)$ & $95(53)$ & $0.944^{\mathrm{b}}$ \\
\hline \multicolumn{2}{c}{ a Independent t test. ${ }^{\mathrm{b}} \chi^{2}$ test. } & & \\
\hline
\end{tabular}

Healthy volunteers without a history of hearing loss or any ear disorders were enrolled as controls. Information on demographic characteristics was collected. Audiometric tests, including pure tone audiometry, tympanometry, stapedial reflexes, auditory brainstem-evoked responses, and computer tomography or magnetic resonance imaging (to exclude acoustic neuroma) were performed in SSNHL patients. The Institutional Review Board of the Kaohsiung Medical University Hospital approved this study (approval No. KMUH-IRB-990243), and informed consent was obtained from all subjects or their guardian if the participant was under 18 years old.

\section{SNP Selection and Genotyping}

Genomic DNA was extracted from peripheral blood using a standard method. Three tSNPs were selected from the HapMap Project (International HapMap Consortium) using the following criteria: (1) a minor allele frequency $\geq 30 \%$ in the Han Chinese population and (2) a linkage disequilibrium value of $\left(\mathrm{r}^{2}\right) \geq 0.8$. The three tSNPs included the nonsynonymous SNP rs2075800 (Glu/ Lys) at exon 2 in the HSPA1L (HSP70-Hom) gene, rs1043618 at the $5^{\prime}$ untranslated region in the HSPA1A (HSP70-1) gene and rs2763979 at 936 bp upstream of the $5^{\prime}$ end of the HSPA1B (HSP70-2) gene.

Genotyping was performed using TaqMan technology (7500 Real Time PCR System, Applied Biosystems, Foster City, Calif., USA), and reactions were performed in 96-well microplates in ABI 9700 thermal cyclers (Applied Biosystems). Fluorescence was measured using an ABI 7500 Real Time PCR System and analyzed with System SDS software version 1.2.3. Each subject was typed for all SNPs.

\section{Statistical Analysis}

Continuous variables were analyzed using independent $t$ tests, and the results are presented as means \pm SD. The allele frequency was obtained using direct gene counting. The Hardy-Weinberg equilibrium was examined in controls using the $\chi^{2}$ test. Multiple logistic regression analysis was performed to adjust for the effects of age and sex while assessing the genetic effects. We examined the effect of the minor allele of each SNP in three genetic models: dominant, additive, and recessive. Linkage disequilibrium was assessed for pairs of SNPs, and haplotype blocks were defined using the default setting in the Haploview software [Barrett et al., 2005]. The Hap-Clustering program [Tzeng et al., 2006] evaluated the haplotype-phenotype association. SPSS 13.0 version for Windows was used for statistical analysis. Two-tailed $p$ values $<0.05$ were considered significant. 
Table 2. Distribution of HSP70 genotype and allele frequencies, ORs, and 95\% CI of SSNHL susceptibility and control groups

\begin{tabular}{|c|c|c|c|c|c|c|c|c|}
\hline \multicolumn{9}{|c|}{ rs2075800 } \\
\hline \multicolumn{9}{|c|}{ Genotypes } \\
\hline $\mathrm{CC}$ & $68(43)$ & $54(30)$ & $122(36)$ & \multirow[t]{2}{*}{0.067} & $1.00^{c}$ & & $1.00^{c}$ & \\
\hline $\mathrm{CT}$ & $74(46)$ & $99(56)$ & $173(51)$ & & $0.59(0.37-0.95)$ & $0.029^{*}$ & $0.59(0.37-0.94)$ & $0.027^{*}$ \\
\hline $\mathrm{C}$ & $210(66)$ & $207(58)$ & $417(62)$ & \multirow[t]{2}{*}{$0.046^{*}$} & \multirow[t]{2}{*}{ NA } & & & \\
\hline $\mathrm{T}$ & $110(34)$ & $149(42)$ & $259(38)$ & & & & & \\
\hline \multicolumn{9}{|c|}{ rs1043618 } \\
\hline \multicolumn{9}{|c|}{ Genotypes } \\
\hline GG & $75(47)$ & $82(46)$ & $157(46)$ & 0.178 & $1.00^{c}$ & & $1.00^{c}$ & \\
\hline $\mathrm{C}$ & $111(35)$ & $114(32)$ & $225(33)$ & 0.463 & NA & & & \\
\hline \multicolumn{9}{|c|}{ rs2763979 } \\
\hline \multicolumn{9}{|c|}{ Genotypes } \\
\hline $\mathrm{CC}$ & $80(50)$ & $101(57)$ & $181(54)$ & \multirow[t]{3}{*}{0.399} & $1.00^{c}$ & & $1.00^{c}$ & \\
\hline $\mathrm{CT}$ & $69(43)$ & $64(36)$ & $133(39)$ & & $1.36(0.87-2.13)$ & 0.179 & $1.37(0.87-2.14)$ & 0.175 \\
\hline TT & $11(7)$ & $13(7)$ & $24(7)$ & & $1.07(0.45-2.51)$ & 0.880 & $1.07(0.46-2.52)$ & 0.878 \\
\hline \multicolumn{9}{|l|}{ Alleles } \\
\hline C & $229(72)$ & $266(75)$ & $495(73)$ & \multirow[t]{2}{*}{0.355} & \multirow[t]{2}{*}{ NA } & & & \\
\hline $\mathrm{T}$ & $91(28)$ & $90(25)$ & $181(27)$ & & & & & \\
\hline
\end{tabular}

${ }^{*} \mathrm{p}<0.05 .{ }^{\mathrm{a}} \chi^{2}$ test. ${ }^{\mathrm{b}}$ Adjusted for age and sex (male, female). ${ }^{\mathrm{c}}$ Reference group.

\section{Results}

\section{Study Participants}

Table 1 presents the baseline characteristics of the subjects. The mean age of the 160 cases ( 86 male and $74 \mathrm{fe}-$ male) was $51.6 \pm 15.0$ years, and the mean age of the 178 control volunteers (95 male and 83 female) was $51.7 \pm 8.0$ years. The sex distribution was 1.2:1 (male to female). The age and sex characteristics were not significantly different between the two groups.

\section{Single SNP Results}

The distribution of HSP70 genotypes was in HardyWeinberg equilibrium among control subjects using $\mathrm{p}=$ 0.05 as the cutoff point. The distributions of genotypes and allele frequencies of the HSP70 SNPs are listed in table 2. The SNP rs2075800 allele T was significantly higher in the control group compared to SSNHL patients ( 42 vs. $34 \% ; \mathrm{p}=0.046$; table 2). None of the SNPs rs1043618 and rs2763979 exhibited significant $p$ values (table 2). The crude and adjusted odds ratios (ORs) were computed for the association analyses between HSP70 genotypes and SSNHL. The multivariate logistic regression model yielded an adjusted OR of 0.59 (95\% confidence interval (CI) $0.37 \sim 0.94 ; \mathrm{p}=0.027$ ) for the genotype CT versus CC of rs2075800 after adjustment for age and sex (table 2). A significant result was observed under the dominant model, which yielded an adjusted OR of $0.59(95 \%$ CI $0.37 \sim 0.92 ; \mathrm{p}=0.019)$ for $\mathrm{T}$ carrier $(\mathrm{TT}+$ CT) versus CC of rs2075800 after adjustment for age and sex (table 3). No significant results for SNPs rs1043618 and rs2763979 were observed in any of the three genetic models.

\section{Linkage Disequilibrium and Haplotype Analysis}

The three SNPs formed one haplotype block. Haplotype analyses demonstrated a global p value of 0.1298 . The distribution of haplotypes between the SSNHL and control groups is listed in table 4. One of these haplotypes, TGC (rs2075800/rs1043618/rs2763979), was signif- 
Table 3. Three SNPs and their relationships with SSNHL under three genetic models (dominant, additive, and recessive)

\begin{tabular}{|c|c|c|c|c|c|c|c|}
\hline SNP & SSNHL & \multicolumn{3}{|c|}{ Genotype (n) } & \multicolumn{3}{|l|}{$\mathrm{p}$ value } \\
\hline HSPA1L & Control & CC (54) & CT (99) & TT (25) & & & \\
\hline rs1043618 & Case & GG (75) & GC (59) & CC (26) & 0.878 & 0.173 & 0.092 \\
\hline HSPA1A & Control & GG (82) & GC (78) & CC (18) & & & \\
\hline
\end{tabular}

Dominant, additive, or recessive model: the rare allele had a dominant, additive, or recessive effect. All p values were adjusted by age and sex. ${ }^{*} \mathrm{p}<0.05$.

Table 4. Results from haplotype analysis which yielded a global $\mathrm{p}$ value of 0.1298

\begin{tabular}{llcl}
\hline Haplotype $^{\mathrm{a}}$ & Case, $\%$ & Control, \% & p value $^{\mathrm{b}}$ \\
\hline CGC & 24 & 18 & 0.1194 \\
CGT & 11 & 8 & 0.2784 \\
CCC & 17 & 15 & 0.5977 \\
CCT & 16 & 17 & 0.9574 \\
TGC & 32 & 42 & $0.0137^{*}$ \\
\hline
\end{tabular}

a The allele order is rs2075800 (HSPA1L)/rs1043618 (HSPA1A)/ rs2763979 (HSPA1B) from left to right. ${ }^{\mathrm{b}}$ Omnibus as the reference group. ${ }^{*} \mathrm{p}<0.05$.

icantly associated with a decreased SSNHL susceptibility compared to all other haplotypes $(\mathrm{p}=0.0137)$. The haplotype TGC (rs2075800/rs1043618/rs2763979) exhibited a frequency of $32 \%$ in cases and $42 \%$ in controls. The other haplotypes did not yield significant results.

\section{Discussion}

We systematically investigated the relationship between three SNPs in the HSP70 gene and SSNHL in the Taiwanese population. Our results revealed that one SNP was associated with SSNHL susceptibility. This significant result stemmed from the HSPA1L (HSP70-Hom; rs2075800) polymorphism of the HSP70 gene, which indicates that the rare $\mathrm{T}$ allele significantly decreases the risk for SSNHL. The logistic regression model yielded an adjusted OR of $0.59(\mathrm{p}=0.027)$ for the genotype CT versus CC of rs2075800 after adjustment for age and sex. Subjects with the heterogeneous CT genotype in our study exhibited a lower incidence of SSNHL than subjects with the homogeneous CC genotype of SNP rs2075800.

The SNP rs2075800 of the HSPA1L (HSP70-Hom) gene is a nonsynonymous variation. Thymine is substituted for cytosine in the HSPA1L polymorphism Glu602Lys $(\mathrm{C}>\mathrm{T}$ transition), which causes a change from glutamic acid to lysine at position 602 in the C-terminal domain of HSP70Hom [Jenkins et al., 2000]. The SNP rs2075800 changes a negatively charged amino acid (Glu) to a positively charged amino acid (Lys). This charge shift may influence the 3D structure of HSP70 protein and altered protein folding. Further studies are needed to address the molecular mechanisms by which the HSP70 gene is involved in the development of SSNHL. The associations of the HSPA1L (HSP70Hom) SNP rs2075800 with the predisposition to some autoimmune diseases has been documented previously [Jenkins et al., 2000; Bogunia-Kubik et al., 2006; Spagnolo et al., 2007]. The HSPA1L (HSP70-Hom) rs2075800 T allele is associated with rheumatoid arthritis [Jenkins et al., 2000], and the HSPA1L SNP rs2075800 C allele is associated with the susceptibility to sarcoidosis and Lofgren's syndrome [Bogunia-Kubik et al., 2006]. A strong association between HSPA1L SNP rs2075800 and the increased risk of uveitis has been reported in patients with sarcoidosis, which suggests that the $\mathrm{C}$ allele of this SNP is a risk factor of sarcoidosis-related uveitis [Spagnolo et al., 2007]. Spagnolo et al. [2007] postulated that the altered expression of HSPA1L influences the ability of the cell to withstand inflammation or alters the apoptotic threshold within the eye. However, SNP rs2075800 has not been associated with hearing impairment. Our previous study revealed an association between HSP70 polymorphism and noise-induced hearing loss in the SNP rs1043618 of gene HSPA1A (HSP701), but not in rs2075800 of HSPA1L (HSP70-Hom) or rs2763979 of HSPA1B (HSP70-2) [Chang et al., 2011]. 
Furthermore, significant results were observed under dominant models in this study, which yielded an adjusted OR of $0.59(\mathrm{p}=0.019)$ for $\mathrm{T}$ carrier $(\mathrm{TT}+\mathrm{CT})$ versus CC of rs2075800 after adjustment for age and sex. This result revealed the tendency for the T-carrier subjects (TT + CT) to exhibit a lower incidence of SSNHL than subjects with the CC genotype. This result indicated that the T carrier is a protective factor for SSNHL.

The multivariate logistic regression model yielded an adjusted OR of 0.57 (95\% CI $0.28 \sim 1.15 ; \mathrm{p}=0.117$ ) for the genotype TT versus CC of rs2075800 after adjustment for age and sex (table 2). However, no significant differences between the SSNHL and control groups at SNP rs2075800 genotype TT versus CC were observed. SNP rs2075800 genotype TT was higher in the control group compared to SSNHL patients (14 vs. $11 \%$; table 2). The small number of subjects in the SSHNL group may have contributed to this result. An increase in the number of SSNHL subjects may reveal a significant difference.

This study analyzed the association of haplotypes with SSNHL susceptibility. Five common haplotypes were identified in our population, and the haplotype TGC (rs2075800/rs1043618/rs2763979) was significantly associated with resistance to SSNHL. The haplotype analysis results provided stronger statistical support for $H S P 70$ as a genetic marker. The current data suggest that HSP70 polymorphisms influence the susceptibility to the development of SSNHL. This investigation is the first report of a potential contribution of the HSP70 genetic variants to the susceptibility of SSNHL.
Three SNPs that are located in 3 genes of the HSP70 family in an Asian population were analyzed. Our results support the association of genetic polymorphisms in the HSP70 genes with an individual's susceptibility to SSNHL. The HSP70 family may play a key role in the development of SSHNL. The major limitation of this study is the small sample size. Further studies to replicate our results are necessary, and further functional analyses of SNP rs2075800 in the HSP70 gene in SSNHL are warranted.

\section{Conclusions}

The results of this study support the influence of genetic polymorphisms of the HSP70 gene on the risk of SSNHL in a Taiwanese population. Furthermore, the T allele of the nonsynonymous SNP rs2075800 may be a protective allele for SSNHL. However, the functional role of SNP rs2075800 in SSNHL requires further investigation.

\section{Acknowledgments}

This work was supported by a grant from the Kaohsiung Medical University Research Foundation (KMU-M100006). The authors thank the Statistical Analysis Laboratory, Department of Medical Research, Kaohsiung Medical University Hospital, Kaohsiung Medical University, for their assistance.

\section{References}

Asea A, Kraeft SK, Kurt-Jones EA, Stevenson MA, Chen LB, Finberg RW, Koo GC, Calderwood SK: HSP70 stimulates cytokine production through a cd14-dependant pathway, demonstrating its dual role as a chaperone and cytokine. Nat Med 2000;6:435-442.

Barrett JC, Fry B, Maller J, Daly MJ: Haploview: analysis and visualization of LD and haplotype maps. Bioinformatics 2005;21:263-265.

-Bogunia-Kubik K, Koscinska K, Suchnicki K, Lange A: HSP70-hom gene single nucleotide $(+2763$ G/A and $+2437 \mathrm{C} / \mathrm{T}$ ) polymorphisms in sarcoidosis. Int J Immunogenet 2006;33:135-140.

-Chang NC, Ho CK, Lin HY, Yu ML, Chien CY, Ho KY: Association of polymorphisms of heat shock protein 70 with susceptibility to noiseinduced hearing loss in the Taiwanese population. Audiol Neurootol 2011;16:168-174.

- Gross M, Eliashar R, Ben-Yaakov A, Ulmansky R, Elidan J: Prevalence and clinical significance of anticardiolipin, anti-beta2-glyco- protein-1, and anti-heat shock protein-70 autoantibodies in sudden sensorineural hearing loss. Audiol Neurootol 2008;13:231-238.

Jenkins SC, March RE, Campbell RD, Milner CM: A novel variant of the MHC-linked hsp70, hsp70-hom, is associated with rheumatoid arthritis. Tissue Antigens 2000;56:38-44.

Macario AJ, Conway de Macario E: Sick chaperones, cellular stress, and disease. N Engl J Med 2005;353:1489-1501.

Matouschek A: Protein unfolding - an important process in vivo? Curr Opin Struct Biol 2003;13:98-109.

Milner CM, Campbell RD: Structure and expression of the three MHC-linked HSP70 genes. Immunogenetics 1990;32:242-251.

- Park SN, Yeo SW, Park KH: Serum heat shock protein 70 and its correlation with clinical characteristics in patients with sudden sensorineural hearing loss. Laryngoscope 2006; 116:121-125.
Schreiber BE, Agrup C, Haskard DO, Luxon LM: Sudden sensorineural hearing loss. Lancet 2010;375:1203-1211.

Spagnolo P, Sato H, Marshall SE, Antoniou KM, Ahmad T, Wells AU, Ahad MA, Lightman S, du Bois RM, Welsh KI: Association between heat shock protein $70 /$ hom genetic polymorphisms and uveitis in patients with sarcoidosis. Invest Ophthalmol Vis Sci 2007;48: 3019-3025.

-Srivastava PK, Udono H, Blachere NE, Li Z: Heat shock proteins transfer peptides during antigen processing and CTL priming. Immunogenetics 1994;39:93-98.

- Tzeng JY, Wang CH, Kao JT, Hsiao CK: Regression-based association analysis with clustered haplotypes through use of genotypes. Am J Hum Genet 2006;78:231-242.

Wu CS, Lin HC, Chao PZ: Sudden sensorineural hearing loss: evidence from Taiwan. Audiol Neurootol 2006;11:151-156. 